\title{
Effects of intensive gait-oriented physiotherapy during early acute phase of stroke
}

\author{
Sinikka H. Peurala, PhD; ${ }^{1-2 *}$ Olavi Airaksinen, MD, PhD; ${ }^{3}$ Pekka Jäkälä, MD, PhD; ${ }^{1}$ Ina M. Tarkka, PhD; \\ Juhani Sivenius, MD, $\mathbf{P h D}^{\mathbf{1 , 4}}$ \\ ${ }^{1}$ Department of Neurology, Kuopio University Hospital, Kuopio, Finland; ${ }^{2}$ Department of Health Sciences, The Finnish \\ Centre for Interdisciplinary Gerontology, University of Jyväskylä, Jyväskylä, Finland; ${ }^{3}$ Department of Physical \\ Medicine and Rehabilitation, Kuopio University Hospital, Kuopio, Finland; ${ }^{4}$ Brain Research and Rehabilitation \\ Center Neuron, Kuopio, Finland
}

\begin{abstract}
We assessed the effects and strenuousness of intensive gait-oriented inpatient rehabilitation initiated very early after stroke. Therapy content and interrater reliability of the assessments were also analyzed. Of 22 patients, 19 (average $8.0 \mathrm{~d}$ poststroke) completed the study. Before rehabilitation, 13 patients were unable to walk or needed two assistants to walk and 6 patients needed one assistant. Patients spent a daily maximum of $1 \mathrm{~h}$ therapy time to obtain 20 min of walking. Additional physiotherapy was also provided during the $3 \mathrm{wk}$ therapy period. Seven structured motor tests were recorded before and after rehabilitation and at 6 months postrehabilitation, and perceived exertion was followed during physiotherapy. After rehabilitation, 16 patients could walk unassisted and 3 needed one assistant to walk. Mean $+/-$ standard deviation exercise walking distance was $10,784+/-4,446 \mathrm{~m}$ and exercise was ranked as slightly strenuous. After $3 \mathrm{wk}$, the patients' $10 \mathrm{~m}$ walking time, ankle spasticity, lower-limb muscle force, and motor scale scores improved $(p<0.05)$. The early intensive rehabilitation was well tolerated and only three patients dropped out. Improved motor abilities were seen in all stroke patients.
\end{abstract}

Clinical Trial Registration: ClinicalTrials.gov; Gait Trainer vs Traditional Physiotherapy in Acute Stroke, NCT00307762; $<\underline{\text { http://clinicaltrials.gov/ct/show/NCT00307762>. }}$.

Key words: acute, body-weight supported gait, cerebral infarction, exercise therapy, neurorehabilitation, physiotherapy, rehabilitation, stroke, task-specific therapy, walking.

\section{INTRODUCTION}

Cerebrovascular stroke is the sixth highest disease burden worldwide in terms of disability-adjusted life years and is the most important cause of severe disability in people living in their own homes. The ability to perform basic activities of daily living (ADL) is reduced initially in three out of four patients with stroke [1]. The most severely affected ADL are transferring, dressing, and walking. The time course and degree of recovery of walking function after stroke and the influence of initial

\footnotetext{
Abbreviations: $6 \mathrm{MWT}=6$-minute walking test, $10 \mathrm{MWT}=$ 10-meter walking test, $\mathrm{ADL}=$ activities of daily living, $\mathrm{BI}=$ Barthel Index, BWS = body-weight support, FAC = Functional Ambulation Category, HR = heart rate, ICC = intraclass correlation coefficient, MAS = Modified Ashworth Scale, MI = Motricity Index, MMAS = Modified Motor Assessment Scale, RMA = Rivermead Motor Assessment, RMA g = RMA gross motor function, RMA 1\&t = RMA leg and trunk, RMI = Rivermead Mobility Index, SD = standard deviation, SSS = Scandinavian Stroke Scale.

*Address all correspondence to Sinikka H. Peurala, PhD; The Finnish Centre for Interdisciplinary Gerontology, University of Jyväskylä, PL35, Jyväskylä 40014, Finland; +358260-2196; fax: +358-260-4600.

Email: sinikka.peurala@sport.jyu.fi

DOI: 10.1682/JRRD.2006.05.0039
} 
lower-limb paresis were studied prospectively in a community-based population of 804 consecutive acute stroke patients in the Copenhagen Stroke Study [2]. The median time from stroke to admission was 12 hours, and the length of hospital stay was 35 days (standard deviation [SD] $=41$ ). The authors reported that from admission to the start of rehabilitation, 51 percent of subjects had no walking function and another 12 percent needed help with walking. Walking function was assessed weekly with the Barthel Index (BI) for walking (no walking function, walking with assistance, or independent walking function) until death or end of inpatient rehabilitation. During hospitalization, 21 percent of the patients died. After inpatient rehabilitation, 22 percent of the remaining patients still had no walking function and 14 percent required assistance. Among those patients with no walking function on admission, 80 percent reached best walking function within 6 weeks and 95 percent within 11 weeks. Of those patients who could initially walk with assistance, 80 percent reached best function within 3 weeks and 95 percent within 5 weeks [2].

Previously published randomized controlled gaitrehabilitation studies report that most patients were 8 to 148 days poststroke when they started rehabilitation intervention; however, the mean starting time was during the late subacute stage [3-7]. Active training might need to be initiated promptly after stroke, i.e., 2 to 8 days poststroke, to promote cortical reorganization and achieve better functional benefits. Several studies established an association between a lower rate of functional recovery and delayed therapy initiation in neurological conditions, and evidence from human studies has shown that intensive rehabilitation should focus on the acute stroke stage [8-9]. The Department of Veterans Affairs/Department of Defense guidelines for stroke rehabilitation recommend that rehabilitation be started as early as possible [9-14]; however, in none of these reports did patients start rehabilitation within 10 days.

Intensive therapy is a complex term that is defined in different ways. In their meta-analysis, Kwakkel et al. presented the effects of the intensity of augmented exercise therapy time on ADL, walking, and dexterity in patients with stroke [15]. The longest daily exercise time found was 100 minutes in Stern et al.'s 1970 study (as cited in Kwakkel et al. [15]) Furthermore, constraint-induced movement therapy to the upper limbs consisted of 6 to 7 hours daily training (e.g., Tarkka et al. [16]). Lowerlimb rehabilitation is more strenuous than upper-limb rehabilitation, which one must consider when planning an intensive program for the lower limbs. Studies also showed that longer duration of lower-limb rehabilitation during the first 20 weeks poststroke led to improved recovery in ADL, walking, and postural control [17]. Longer duration of lower-limb rehabilitation also resulted in faster comfortable walking speed compared with either longer rehabilitation sessions for the paretic upper limbs or a control treatment [17-18]. Longer duration of upper-limb rehabilitation benefited only dexterity compared with the control group. We provided 20 minutes daily of gait exercises within 1 hour plus 55 minutes of other gait-oriented rehabilitation, for a total of $115 \mathrm{~min}-$ utes of daily training over 3 weeks. We believe that an early start, active and focused therapy, and long training time can be achieved at the acute stage of stroke.

This study assessed the effects and strenuousness of intensive gait-oriented rehabilitation in patients with acute stroke. Patients entered the study as soon as possible but at least within 10 days after their stroke. The effects of rehabilitation were followed up at 6 months. In addition, we analyzed the therapy content in detail to clearly illustrate the actual activities engaged in instead of merely naming a physiotherapy approach. We used numerous structured tests with rating scales and thus also analyzed interrater reliability.

\section{METHODS}

\section{Subjects}

Twenty-two patients with acute stroke participated in this trial (Table 1). On admission, they were diagnosed with either a first supratentorial stroke or no significant disturbance from an earlier stroke (Modified Ranking Scale 0-2) [19] and were selected for this study if they had (1) Functional Ambulation Category (FAC) of 0-3 [20], (2) voluntary movement on the leg of the affected side, (3) BI score of 25-75 [21], (4) age 18 to 85 years, (5) no unstable cardiovascular disease, (6) body mass index <32, (7) no severe malposition of joints, and (8) no severe cognitive or communicative disorders. All patients were initially diagnosed by magnetic resonance imaging or computerized tomography. Patients who fulfilled the criteria in the neurological examination, which was performed within 10 days of stroke onset, and who provided written informed consent were randomly allocated into one of two intensive walking exercise groups and 
Table 1.

Characteristics of patients with acute stroke $(N=22)$.

\begin{tabular}{|c|c|c|c|c|}
\hline \multirow{2}{*}{ Variable } & \multicolumn{2}{|c|}{ Completed Rehabilitation $(n=19)$} & \multicolumn{2}{|c|}{ Dropped Out $(n=3)$} \\
\hline & Mean \pm SD & Range & Mean \pm SD & Range \\
\hline$\overline{\text { Age (yr) }}$ & $66.4 \pm 10.3$ & $43-80$ & $72.0 \pm 13.0$ & $59-84$ \\
\hline Poststroke (d) & $8.0 \pm 3.3$ & $3-17$ & $4.6 \pm 4.4$ & $1-10$ \\
\hline Body Mass Index & $27.1 \pm 4.1$ & 21.7-39.6 & $27.4 \pm 4.4$ & $23.6-32.2$ \\
\hline Scandinavian Stroke Scale (0-48 points) & $36.2 \pm 8.5$ & $21-51$ & $39.7 \pm 7.6$ & $33-48$ \\
\hline \multirow[t]{2}{*}{ Barthel Index (0-100 points) } & $39.7 \pm 15.0$ & $0-70$ & $63.3 \pm 27.5$ & $35-90$ \\
\hline & \multicolumn{2}{|c|}{ Frequency } & \multicolumn{2}{|c|}{ Frequency } \\
\hline Male/Female & \multicolumn{2}{|c|}{$10 / 9$} & \multicolumn{2}{|c|}{$1 / 2$} \\
\hline Infarction/Hemorrhage & \multicolumn{2}{|c|}{$16 / 3$} & \multicolumn{2}{|c|}{$3 / 0$} \\
\hline Left/Right Hemiparesis & \multicolumn{2}{|c|}{$12 / 7$} & \multicolumn{2}{|c|}{$1 / 2$} \\
\hline No/Yes Aphasia & \multicolumn{2}{|c|}{$13 / 6$} & \multicolumn{2}{|c|}{$3 / 0$} \\
\hline No/Yes Neglect & \multicolumn{2}{|c|}{$16 / 3$} & \multicolumn{2}{|c|}{$3 / 0$} \\
\hline Normal/Abnormal Position Sense & \multicolumn{2}{|c|}{$11 / 7$} & \multicolumn{2}{|c|}{$2 / 1$} \\
\hline \multicolumn{5}{|l|}{ Functional Ambulatory Category ${ }^{*}$} \\
\hline 0 & \multicolumn{2}{|c|}{13} & \multicolumn{2}{|c|}{2} \\
\hline 1 & \multicolumn{2}{|c|}{5} & \multicolumn{2}{|c|}{0} \\
\hline 2 & \multicolumn{2}{|c|}{1} & \multicolumn{2}{|c|}{1} \\
\hline
\end{tabular}

${ }^{*}$ Functional Ambulatory Category: 0 = unable to walk or needs two assistants, 1 = needs someone to support continuously while walking to shift weight or maintain balance, 2 = needs someone to support continuously/occasionally while walking to maintain balance and coordination.

$\mathrm{SD}=$ standard deviation.

received 3 weeks of inpatient rehabilitation in the acutecare hospital. However, we do not report the separate groups here. We are still recruiting patients, and our present focus was to elucidate the changes in motor ability in the pooled data of all the patients. The ethical committee of Kuopio University Hospital (Kuopio, Finland) approved the study.

A neurologist used the Scandinavian Stroke Scale (SSS) [22] and the BI [21] to assess the functional status of the 22 patients with acute stroke. The SSS contains items on consciousness; orientation; eye movements; facial palsy; motor function of the arm, hand, and leg; gait; and speech. Each item is scored from 0-12 and the maximum score is 48 (Table $\mathbf{1}$ ). The BI contains items on feeding, moving from and to a wheelchair, personal toileting, bathing self, walking on a level surface, ascending and descending stairs, dressing, and bowel and bladder control. Each item is scored from $0-15$ and the maximum score is 100 (Table 1). Thirteen of the patients had normal position sense in the ankle on the hemiparetic side (Table 1). One patient's position sense could not be assessed because of aphasia. Position sense was measured with the patient supine and an observer moving the paretic ankle in different directions. The patient copied the movements with the unaffected leg. Alternatively, the observer moved the paretic ankle and asked the patient to identify the direction of movement. Fifteen of the patients could not walk or needed two assistants to help them walk $(\mathrm{FAC}=0)$. Five patients needed the constant attention of one assistant for walking $(\mathrm{FAC}=1)$. Two patients needed someone for balance support $(\mathrm{FAC}=2)$. None of the patients was in the more independent walking categories of FAC 3, 4, or 5 (Table 1). Unfortunately, one patient did not fulfill our inclusion criteria within 10 days of her stroke and thus her program was delayed until 17 days poststroke.

\section{Intervention}

The objective of our 3-week inpatient rehabilitation for acute patients was to enhance their motor abilities and help them recover walking independence as soon as possible. Every day for 3 weeks, each patient spent a maximum of 1 hour to obtain 20 minutes of actual walking either on the electromechanical gait trainer (Gait Trainer ${ }^{\circledR}$; Reha-Stim, Berlin, Germany, Figure 1) or overground (Figure 2). Each 


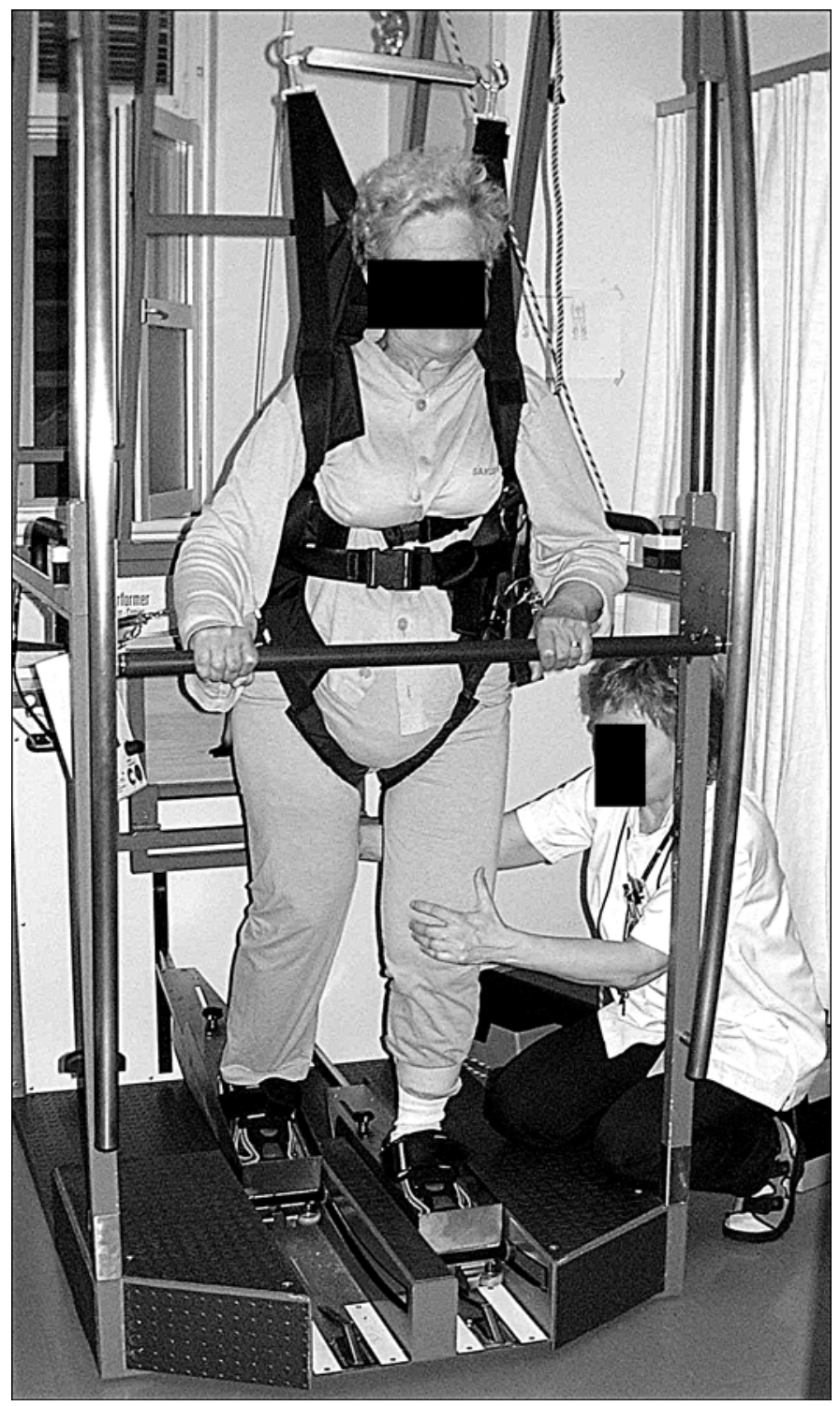

Figure 1.

Patient with acute stroke walking on electromechanical Gait Trainer ${ }^{\circledR}$ (Reha-Stim, Berlin, Germany) with minimal assistance from physiotherapist.

patient also received other gait-oriented physiotherapy for 55 minutes each day. These additional physiotherapy sessions were carried out according to individually set goals.

In the gait trainer, the patient was supported with a harness and his or her feet were placed on motor-driven footplates. A speed up to $2 \mathrm{~km} / \mathrm{h}$ was selected. The amount of body-weight support (BWS) provided by the harness was chosen according to the patient's individual

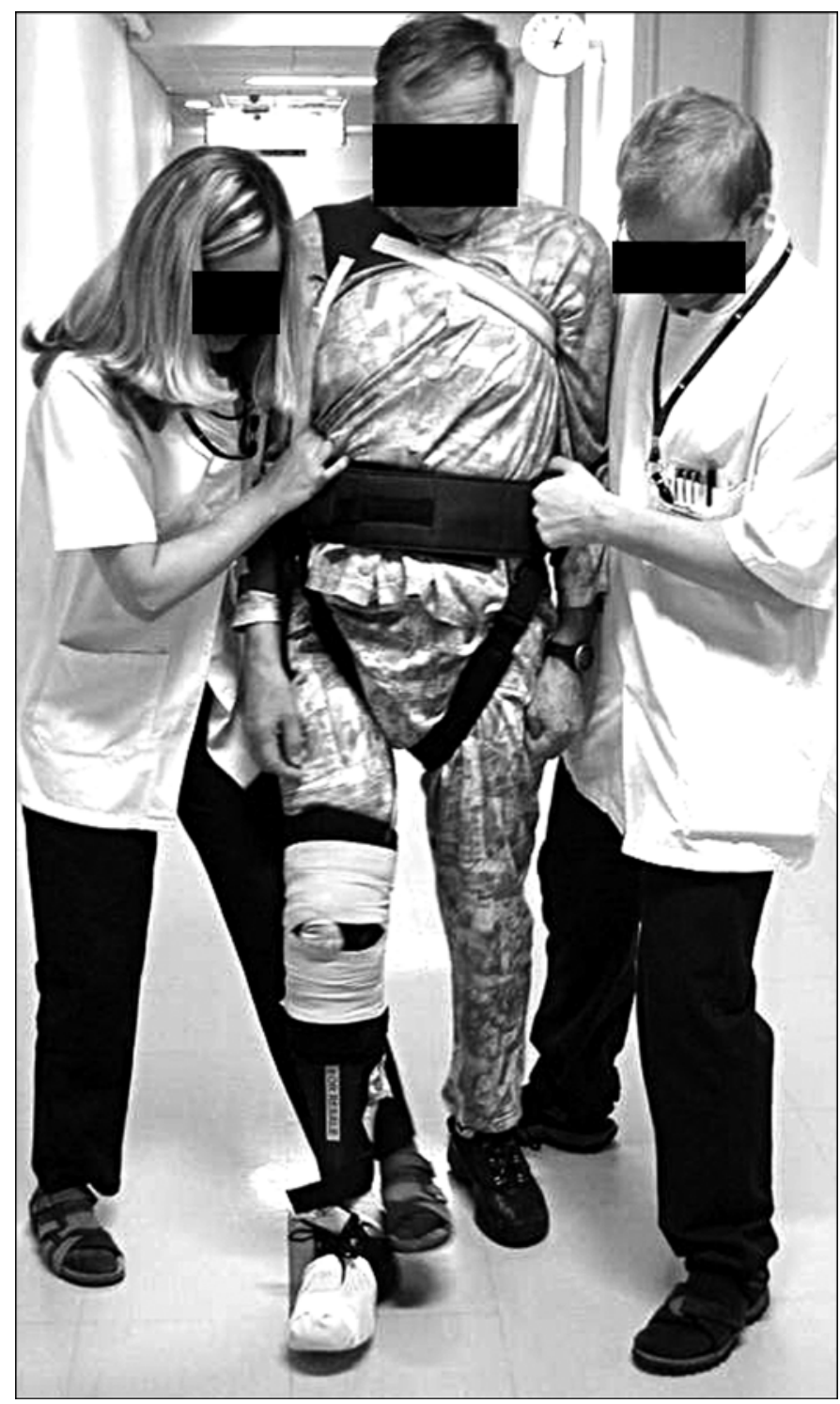

Figure 2.

Patient with acute stroke walking overground with moderate assistance from two physiotherapists, walking belt, knee orthosis, and elastic bandage.

needs. The gait trainer measures the support given by the harness in kilograms. When a patient's body weight is known, the percentage support of body weight can be calculated. We progressed training by increasing the speed of the gait trainer and aiming for a BWS of $<20$ percent body weight [23-24]. Reducing the BWS as the training progresses is important for effective and progressive activation of the lower-limb muscles and increased energy expenditure [25-27]. Previous studies have indicated that 
retraining gait with BWS leads to successful recovery of walking in patients with stroke and that progressively decreasing BWS improves walking more effectively $[3,28]$. The participants who did not practice with the gait trainer practiced walking overground with one or two physiotherapists and individual walking aids. We progressed their training by decreasing the amount of manual guidance and reliance on walking aids. All patients were guided verbally and/or manually.

\section{Assessments}

\section{Training}

The patients evaluated their efforts using the Borg Rating of Perceived Exertion Scale (from 6-20; e.g., 7 = very marginally strenuous, 19 = extremely strenuous,
(Table 2) [29]. Ratings were recorded each session during the last minute of the 20-minute walking exercise and during the last minute of the other physiotherapy session. Heart rate (HR) was recorded with a heart-rate monitor (Polar ${ }^{\circledR}$; Polar-electro Oy, Kempele, Finland). We continuously monitored HR during the 20-minute walking exercise and recorded the data for the last minute.

The physiotherapist recorded the daily content and duration of the additional physiotherapy (Table 3). During the 20-minute walking exercises on the gait trainer, the speed, session duration, number of steps and distance, and amount of BWS were recorded. For those who walked overground, the session duration, distance walked, and use of walking aids were recorded. We allowed each patient a daily maximum of 1 hour to achieve the target of 20 minutes real walking time.

Table 2.

Outcome measures used in this study.

\begin{tabular}{ll}
\multicolumn{1}{c}{ Parameter } & Item Assessed \\
\hline 10-Meter Walking Test (s) & Walking speed \\
6-Minute Walking Test (m) & Walking endurance \\
Modified Ashworth Scale (Category 0-5) & Spasticity \\
Motricity Index (Category 0-5) & Muscle force \\
Modified Motor Assessment Scale (0-48 points) & Motor ability \\
Rivermead Motor Assessment & Gross motor ability \\
Gross Motor Function (0-13 points) & Leg and trunk function \\
Leg \& Trunk Function (0-10 points) & Perceived motor ability \\
Rivermead Mobility Index (0-15 points) & Perceived exertion \\
Borg Rating of Perceived Exertion Scale (6-20 points) & Exertion \\
Heart Rate & \\
\hline
\end{tabular}

Table 3.

Duration of supplemental physiotherapy by specific therapeutic mode. During 3-week period, acute stroke patients $(n=19)$ received physiotherapy in addition to daily 20-minute gait training.

\begin{tabular}{lccc}
\hline \multicolumn{1}{c}{ Therapeutic Mode } & Patients (No.) & Duration (min) & Range \\
\cline { 2 - 4 } \multicolumn{1}{c}{ Tonus Inhibition } & 12 & Mean \pm SD & $0-105$ \\
Stretching & 13 & $27 \pm 34$ & $0-130$ \\
Soft Tissue Techniques & 5 & $37 \pm 36$ & $0-15$ \\
Exercises in Lower Initial Positions & 19 & $151 \pm 51$ & $60-220$ \\
Upper-Limb/Trunk Exercises While Sitting & 19 & $101 \pm 69$ & $25-270$ \\
Hand Exercises & 10 & $18 \pm 35$ & $0-120$ \\
Lower-Limb Exercises While Sitting & 17 & $44 \pm 26$ & $0-95$ \\
Transfer Exercises & 18 & $55 \pm 38$ & $0-105$ \\
Exercises While Standing & 19 & $156 \pm 59$ & $30-230$ \\
Gait on Even Floor & 19 & $96 \pm 47$ & $25-165$ \\
Stairs & 19 & $99 \pm 52$ & $20-200$ \\
Gait on Uneven Ground & 11 & $26 \pm 35$ & $0-100$ \\
Time for Evaluation/Planning & 4 & $2 \pm 13$ & $0-45$ \\
Total & - & $815 \pm 16$ & - \\
\hline SD $=$ standard deviation. & & \\
\hline
\end{tabular}




\section{Outcomes}

We assessed the efficacy of our 3-week physiotherapy program by the patients' 10-meter walking test (10MWT) [30], 6-minute walking test (6MWT) [31], spasticity, muscle force, and motor ability outcomes (Table 2). In the 10MWT, patients were asked to walk as quickly as possible. In the 6MWT, patients were asked to walk $30 \mathrm{~m}$ back and forth as quickly as possible while pacing themselves so that they could complete the task. In both walking tests, patients were allowed to use an orthosis. Partial support was allowed such that a physiotherapist could, for example, hold the walking belt during a test but not push forward or move the patient's legs. We assessed spasticity of the paretic leg with the Modified Ashworth Scale (MAS) (scored from $0=$ no increase in muscle tone to 5 = affected part rigid in flexion or extension) [30]. We used the Motricity Index (MI) to test muscle force [32], including hip flexors, knee extensors, and ankle dorsiflexors (scored from $0=$ no movement to $5=$ full range of movement against power and same force as the opposite side). We assessed each patient's motor abilities with the Modified Motor Assessment Scale (MMAS) [33], Rivermead Motor Assessment (RMA) scale [30], and Rivermead Mobility Index (RMI) [32]. The MMAS items assessed were supine to lying on side, supine to sitting, balanced sitting, sitting to standing, walking, upper-arm function, hand movements, and advanced hand activities (scored from 0-6, maximum of 48). Two of the three RMA sections were used: gross motor function (RMA g) and leg function and trunk control (RMA l\&t). Within the RMA g, we tested 13 items, including sitting, transfers, walking, climbing, running, and hopping. Within the RMA l\&t, we tested in hierarchical order 10 items, including rolling to the affected and unaffected sides, bridging, sit-stand-transfer, lifting the affected leg over the side of bed, stepping, foot-tapping, voluntary dorsiflexion with flexed and extended leg, and selective knee flexion while standing with the hip in a neutral position. Performance of each item scored 1 point. The testing was discontinued if the patient was unable to perform two consecutive items. The 15-item RMI was similarly completed, except that we obtained RMI scores by asking the patient if he or she could perform the particular activities.

A physiotherapist and an independent observer simultaneously assessed patients at the start, after 2 weeks, and at the end of the 3 weeks of rehabilitation to calculate the interrater reliability of the measures. The independent observer did not know the type of exercise practiced by the patient. All measurements were also performed at 6 months as a follow-up.

\section{Statistical Analysis}

We performed statistical analyses with SPSS 11.0 (SPSS, Inc, Chicago, Illinois). We calculated descriptive statistics for age, time since stroke onset, body mass index, HR, SSS, BI, total distance (in meters) walked in 15 gait sessions, mean values of speed and amount of BWS in the gait trainer, Borg Rating of Perceived Exertion Scale ratings, and the content of the additional physiotherapy provided. Sex, diagnosis, side of hemiparesis, aphasia, neglect, and FAC were considered in the statistics.

We computed interrater reliability for the MAS [30], MI [32], MMAS [33], both RMA sections [32], and RMI [31] and obtained intraclass correlation coefficients (ICCs) or Kendall $\tau$ b rank correlation coefficients (for categorical parameters).

To evaluate the effect of rehabilitation, we tested the normal distributions of the results with the KolmogorovSmirnov test. First, we restructured walking test data to account for those patients who were unable to walk at the beginning of rehabilitation. Analysis of repeated measures data (within and between factors) was used to evaluate changes from the beginning to the end of rehabilitation (Bonferroni adjustments were performed). We sought to elucidate the changes in the motor ability of the pooled 19 patients. The interactions and group differences (walking either in the electromechanical gait trainer or overground) were not statistically significant. We used Friedman tests to evaluate the changes from the start to the end of rehabilitation in the nonparametric variables. When differences were found, we performed the Wilcoxon signed rank test. For paired samples, we used a $t$-test to determine whether the patients' results at the end of the 3 weeks and at the 6-month follow-up were similar. Results were significant at $p<0.05$.

\section{RESULTS}

\section{Exercise Strenuousness and Intensity}

Nineteen of twenty-two patients completed the rehabilitation period. One patient withdrew from the study after five sessions because of scheduling problems, and two patients withdrew because they felt that the protocol was too demanding. The maximum real walking time during the 3 weeks was 300 minutes (total treatment time 
allowed $=900$ minutes), and the mean walking time was 291 minutes (range 234-300 minutes). Over that time, the patients walked a mean \pm SD distance of 10,784 \pm 4,446 $\mathrm{m}$. In the additional physiotherapy, the patients walked a mean of 815 minutes (range 770-825 minutes, maximum 825 minutes) (Table 3 ). Mean \pm SD perceived exertion in the walking exercises was $14.9 \pm 1$ on the Borg Rating of Perceived Exertion Scale. The mean \pm SD HR during the last minute of the 20-minute walking exercise was $103.6 \pm 17$ beats $/ \mathrm{min}$.

At the start of rehabilitation, the mean \pm SD speed on the gait trainer was $1.5 \pm 0.2 \mathrm{~km} / \mathrm{h}$; by the end of rehabilitation, it was $2.0 \pm 0.3 \mathrm{~km} / \mathrm{h}$. At the start, mean $\pm \mathrm{SD}$ BWS on the gait trainer was $45.0 \pm 32$ percent body weight; at the end, this value had declined to $7.6 \pm 12$ percent body weight. Patients walking overground usually needed two physiotherapists to assist them at the beginning. Later, manual guidance by one physiotherapist was sufficient. A walking belt was used by half the patients who walked overground. Once patients could increase their walking speed, a knee orthosis was often used to prevent knee overextension. Occasionally, a long limb brace was used instead of a knee orthosis. A peroneal orthosis or an elastic bandage was used in most of the patients who walked overground. Most of the supplementary physiotherapy sessions included three therapy modes: exercises in lower initial positions, seated arm or trunk exercises, and standing exercises (Table 3). The patients' mean \pm SD exertion during the supplementary physiotherapy was $14.5 \pm 1$ on the Borg Rating of Perceived Exertion Scale.

\section{Reliability Assessments}

At the beginning of rehabilitation, two people (a physiotherapist and an independent observer) assessed 21 patients; at the second and third assessments, these same two people assessed 16 patients. The ICC values for the MMAS, MI, RMA sections, and RMI ranged from 0.98 to 1.00 (Table 4). The Kendall $\tau \mathrm{b}$ for the MAS were 0.43 to 1.00 hip, 0.41 to 0.61 knee, and 0.32 to 0.56 ankle; at the start of rehabilitation, the hip and ankle evaluations differed significantly $(p=0.02)$ (Table 4).

\section{Effects of Rehabilitation}

The motor abilities of the pooled 19 patients with acute stroke significantly improved during the 3 weeks of gait-oriented rehabilitation. The 10MWT score decreased by 53 percent $(p=0.04)$ and the MMAS score increased by 87 percent $(p<0.001$, Table 5). The RMA g, RMA l\&t, and RMI scores more than doubled ( $p<0.001$, Table $5)$. The 6MWT did not change significantly $(p=0.09$, Table 5). Ankle ( $p=0.01)$, but not knee $(p=0.55)$ or hip $(p=0.07)$, spasticity decreased during the rehabilitation. The median ankle, knee, and hip spasticity were scored as 0 on the MAS throughout rehabilitation. Ankle dorsiflexion force increased, as did knee flexion and hip flexion force $(p<0.001)$. Median ankle dorsiflexion and knee flexion muscle force were 2 on the MI. The median muscle

Table 4.

Kendall $\tau$ b and $p$-values for Modified Ashworth Scale (MAS) and Motricity Index (MI), and intraclass correlation coefficients (ICCs) and 95\% confidence intervals (CIs) for Modified Motor Assessment Scale (MMAS), Rivermead Motor Assessment Scale gross motor function (RMA g), RMA leg and trunk (RMA l\&t), and Rivermead Mobility Index (RMI) for patients with acute stroke, as assessed by physiotherapist and researcher at start, after 2 weeks, and at end of 3 weeks of rehabilitation.

\begin{tabular}{|c|c|c|c|c|c|c|}
\hline \multirow{2}{*}{ Outcome Variables } & \multicolumn{2}{|c|}{ At Start } & \multicolumn{2}{|c|}{2 Weeks } & \multicolumn{2}{|c|}{3 Weeks } \\
\hline & Kendall $\tau \mathbf{b}$ & $p$-Value & Kendall $\tau \mathbf{b}$ & $p$-Value & Kendall $\tau \mathbf{b}$ & $p$-Value \\
\hline MAS Hip & 0.60 & 0.02 & 0.43 & 0.32 & 1.00 & 0.08 \\
\hline MAS Knee & 0.41 & 0.17 & 0.54 & 0.27 & 0.61 & 0.27 \\
\hline MAS Ankle & 0.56 & 0.02 & 0.54 & 0.27 & 0.32 & 0.25 \\
\hline MI Hip Flexors & 0.96 & $<0.001$ & 0.91 & $<0.001$ & 0.94 & $<0.001$ \\
\hline MI Knee Dorsiflexors & 0.82 & $<0.001$ & 0.69 & $<0.001$ & 0.60 & 0.006 \\
\hline MI Ankle Flexors & 0.99 & $<0.001$ & 0.94 & $<0.001$ & 0.93 & $<0.001$ \\
\hline & ICC & $95 \%$ CI & ICC & $95 \%$ CI & ICC & $95 \%$ CI \\
\hline MMAS & 1.00 & $1.00-1.00$ & 1.00 & $1.00-1.00$ & 0.99 & $0.99-1.00$ \\
\hline RMA g & 1.00 & $1.00-1.00$ & 0.98 & $0.95-0.99$ & 0.99 & $0.98-1.00$ \\
\hline RMA 1\&t & 0.99 & $0.98-1.00$ & 0.99 & $0.96-1.00$ & 0.98 & $0.95-0.99$ \\
\hline RMI & 0.99 & $0.98-1.00$ & 1.00 & $1.00-1.00$ & 1.00 & $0.99-1.00$ \\
\hline
\end{tabular}


JRRD, Volume 44, Number 5, 2007

Table 5.

Gait and motor task performance of 19 patients with acute stroke at start, after 2 weeks, and at end of 3 weeks of rehabilitation.

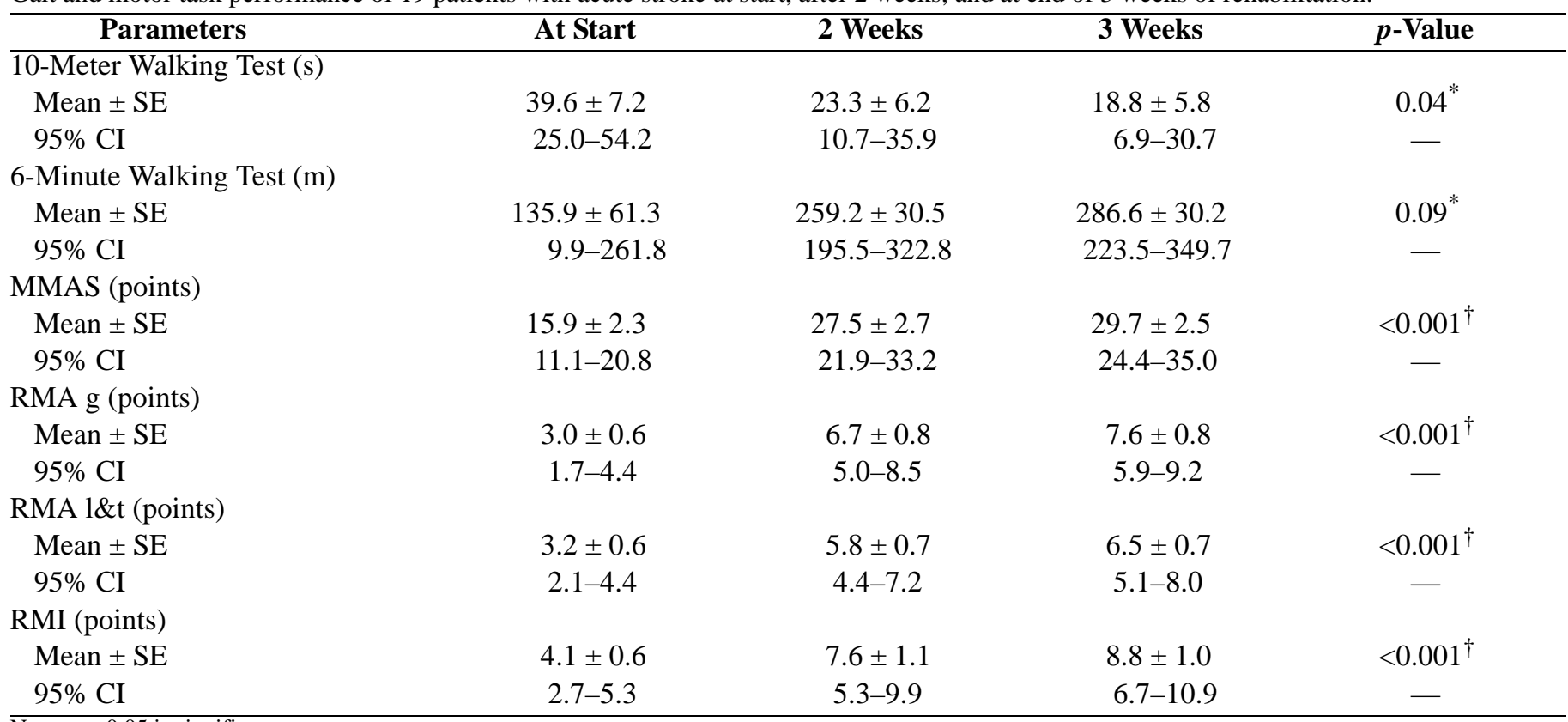

Note: $p<0.05$ is significant.

*Analysis of repeated measures of reconstructed data.

${ }^{\dagger}$ Analysis of repeated measures.

$\mathrm{CI}=$ confidence interval, MMAS $=$ Modified Motor Assessment Scale (maximum 48 points), RMA g = Rivermead Motor Assessment gross motor function (maximum 13 points), RMA 1\&t = RMA leg \& trunk function (maximum 10 points), RMI = Rivermead Mobility Index (maximum 15 points), SE = standard error.

force of the hip flexors was 2 at the start of rehabilitation and at 2 weeks but had risen to 3 by the end of rehabilitation.

\section{Long-Term Effects}

Seventeen patients were available for the 6-month follow-up assessment (Table 6). The 10MWT $(p=0.90)$, the 6MWT ( $p=0.75)$, and MMAS $(p=0.12)$ did not change significantly between the end of rehabilitation and follow-up. Although the 10MWT did not change, three patients who could not walk the 10MWT at the end of rehabilitation could perform this task at follow-up. These three patients' walking times were 35,63 , and $180 \mathrm{~s}$, respectively. Another three patients who could not complete the 6MWT at the end of rehabilitation could do so at the follow-up. Their respective walking distances were 13, 98, and $117 \mathrm{~m}$. Two patients remained unable to perform the 6MWT at the follow-up.

The RMA g increased by 3 points $(p<0.001)$, the RMA l\&t increased by 1 point ( $p=0.04)$, and the RMI increased by 3 points $(p<0.001)$ at the follow-up (Table 6 ).

\section{DISCUSSION}

Intensive walking exercise with additional physiotherapy during an early stage after stroke was well tolerated. Patients achieved major improvements in motor abilities during the first month after stroke. The improvements remained at the 6-month follow-up and certain motor abilities had continued to improve. At the beginning of the intensive rehabilitation, 13 of the 19 patients could not walk or needed two therapists to assist them $(\mathrm{FAC}=0)$ and none could walk without some manual assistance. Nine patients could complete the 10MWT (three with partial support) and three patients could complete the 6MWT at the beginning of rehabilitation. By the end of the rehabilitation period, 16 patients could complete the 10MWT and 14 patients the 6MWT. After we restructured the walking test data to account for the patients who were unable to walk at the beginning of rehabilitation, the statistics showed major improvements in the 10MWT times. The 6MWT scores tended to increase, but the widely variable results and small number of patients able to perform the 6MWT at the beginning nullified any statistical evaluation. All 17 patients available 
Table 6.

Gait and motor task performance (mean \pm standard error) of 17 patients with acute stroke at end of 3-week rehabilitation period and at 6-month follow-up.

\begin{tabular}{lcccc}
\hline \multicolumn{1}{c}{ Parameter } & $\boldsymbol{n}$ & 3 Weeks & 6 Months & $\boldsymbol{p}_{\text {-Value* }}$ \\
\hline 10-Meter Walking Test (s) & 14 & $17.0 \pm 2.5$ & $17.1 \pm 2.8$ & 0.90 \\
6-Minute Walking Test (m) & 12 & $324.8 \pm 33.1$ & $314.0 \pm 35.0$ & 0.75 \\
MMAS (points) & 17 & $30.1 \pm 2.6$ & $32.7 \pm 2.3$ & 0.12 \\
RMA g (points) & 17 & $7.5 \pm 0.9$ & $10.4 \pm 0.6$ & $<0.001$ \\
RMA l\&t (points) & 17 & $6.8 \pm 0.7$ & $7.6 \pm 0.6$ & 0.04 \\
RMI (points) & 17 & $8.9 \pm 1.0$ & $11.8 \pm 0.6$ & $<0.001$
\end{tabular}

Note: $p<0.05$ is significant.

${ }^{*} t$-test for paired sample.

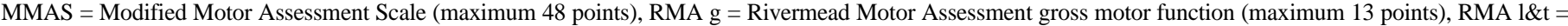
RMA leg \& trunk function (maximum 10 points), RMI = Rivermead Mobility Index (maximum 15 points).

at the 6-month follow-up could walk the 10MWT and only 2 could not complete the 6MWT at this point. Statistics from those who had walking test values at the end of rehabilitation showed stable improvements at the followup. Overall, the walking speed achieved by 6 months after the stroke was about $0.6 \mathrm{~m} / \mathrm{s}$ and the walking distance in 6 minutes was slightly more than $300 \mathrm{~m}$. However, a wide variety existed between the patients.

In addition to our patients' poor initial walking abilities, the BI was about 40 percent of the maximum possible score at the beginning of rehabilitation. Likewise, the motor ability test scores (the mean MMAS score of 15.9 out of 48, the RMA g of 3.0 out of 13, the RMA I\&t of 3.2 out of 10 , and the RMI of 4.1 out of 15 ) reflected the patients' poor motor abilities before rehabilitation. In the MMAS subitems supine to lying on side, supine to sitting, balanced sitting, sitting to standing, and walking, the mean scores ranged from 0.63 to 4.37 out of the maximum subscore of 6 points; in the upper-arm function, hand movement, and advanced hand activity subitems, the mean scores ranged from 0.84 to 1.95 . The motor-ability test scores more than doubled during rehabilitation. Nevertheless, the total end motor-ability test scores were 50 to 76 percent of maximum, depending on the test. The muscle power in the paretic lower limb indicated that movements were possible but not in the full range. The muscle power and motor ability tests showed good to excellent reliability based on the Kendall $\tau$ b or ICC; a coefficient $>0.85$ is required for excellent reliability [34].

The spasticity scale (MAS) presented poor reliability, with a low Kendall $\tau$ b, and the results of the MAS need to be viewed cautiously.

Despite poor motor ability at the beginning, 19 of the 22 patients practiced intensively during the 3 weeks.
Three patients dropped out: one because of scheduling problems and two because they found the protocol too demanding. Overall, the patients evaluated both the walking exercises and additional physiotherapy as either slightly strenuous or strenuous, but not as very strenuous. Their HRs were close to $100 \mathrm{bpm}$ during the last minute of the walking exercises, which is in line with their perceived exertion. One must consider that beta-blockers might have lowered the HR in some patients.

The more seriously affected the patients were, the more support they needed. This fact was reflected in the large amount of manual guidance and number of orthotic devices used during overground walking exercises. In the gait trainer, manual guidance was needed mostly during transfer to and from the device. The limitations of our study are that we did not have a structured questionnaire to survey the physiotherapists about their efforts in the gait training or any structured recording of the time spent to achieve the 20 minutes of walking exercise, except for the limit of 60 minutes in both groups. The physiotherapists believed that the patients using the gait trainer required less time to achieve 20 minutes than those walking overground.

Our study is one of the first clinical trials to provide intensive gait rehabilitation at such an early stage after stroke. In previous randomized controlled trials, gait rehabilitation was not started until 8 days poststroke (range 8-56 days) [5-6]. Thus, patients were usually in a more subacute than acute phase, e.g., 27-148 days [3] or 4044 days [4,7]. In a randomized controlled study of 27 patients, the inclusion criteria for rehabilitation stated that the patient had to be $0-7$ days poststroke but, in fact, the times were 8-13 days [34]. In that study, the time dedicated to gait training was the most important factor 
in improving gait ability and gait velocity among three different therapy groups. The mean total time of gait training was from 28 to 37 percent of the mean total rehabilitation time in the different groups over a 5-week period. In our study, we devoted 46 percent of the time to gait training over a 3-week period. If we were to include the amount of standing exercises done by our patients, total treatment time in an upright position would have comprised 60 percent of the 18 hours of training over the 3-week period.

The duration of daily physiotherapy in our study was more than in most of the studies cited by Kwakkel et al. [15], who presented the effects of the intensity of augmented exercise therapy time on ADL, walking, and dexterity in patients with stroke. In only one study cited in Kwakkel et al. [15], Stern et al., did the patients receive more than 75 minutes of exercise daily (100 minutes in the intervention group). In our study, real walking time was distinguished from training time. For example, in the 20-minute walking exercise, 20 minutes was the real walking time but up to 1 hour was allowed to achieve this. Possible rests during walking exercises were omitted. Thus, the amount of time spent daily with a physiotherapist was always at least 75 minutes and sometimes as much as 115 minutes.

In previous studies, the content of supplementary physiotherapy was poorly described. Nilsson et al. [5], for instance, aimed to improve motor control and strengthen functionally weak muscles. They used transfers and various motion exercises and techniques to improve motor function in the paretic side. In Kosak and Reding's study [4], physiotherapy sessions were functionally oriented, incorporated various motor facilitation and motor control techniques, and often included bracing and walking assistive devices. In the present study, we analyzed the content of the patients' active practice (Table 3).

Intensive rehabilitation during the acute stage of stroke may enhance the functional outcome. Kwakkel et al. studied the intensity of upper- and lower-limb training from the very early stage of stroke onward during a 20-week inpatient rehabilitation period [17]. The 45 minutes of lower-limb training and 15 minutes of upper-limb training every weekday resulted in better ADL scores (BI), walking velocity (10MWT), and hand skills (Action Research Arm test) than were achieved by 15 minutes of training each of the lower and upper limbs. Patients in the more intensive lower-limb training group also reached a higher ADL level earlier than the group with 45 minutes of upper and 15 minutes of lower-limb training or the group with less intensive training.

Conventionally, the rehabilitation that patients receive during the early acute-care stage of stroke is not as intensive as was in the present study. They may only stay a few days in the acute-care hospital before being transferred to a health center or elsewhere. Differences likely exist between countries in the length of stay in the acute-care hospital. In our study, the patients received intensive rehabilitation during acute care. Ethically, we could not analyze a group of patients without rehabilitation. Unfortunately, we were unable to test a corresponding conventional rehabilitation group with the same tests.

Our intensive strategies led to a satisfactory level of motor ability in selected patients with acute stroke at 4 weeks after stroke onset. The motor ability of our patients was better than that described in other studies in which patients with stroke started their rehabilitation during the subacute phase. For example, in Visintin et al.'s study, the patients' 6MWT (27-148 days poststroke) was about $45 \mathrm{~m}$ at the beginning of rehabilitation [3]. In our study (22-38 days poststroke, after rehabilitation), the $6 \mathrm{MWT}$ was $287 \pm 30 \mathrm{~m}$. We would be interested to know whether these differences are attributable to the delayed start of training in the Visintin et al. study. In Richards et al.'s study [34], the patients, like our patients, started rehabilitation early. However, their patients' walking velocity was $0.31 \mathrm{~m} / \mathrm{s}$ in the most intensive group at 6 weeks, whereas our patients' was already $0.53 \mathrm{~m} / \mathrm{s}$ at 3 weeks. High variability is known to exist in the functional abilities of patients with stroke, but whether the differences are because of the more intensive gait training provided in our study would be interesting to know. Further studies must clarify whether early intensive gait rehabilitation leads not only to faster improvement but also to permanently better walking outcomes.

\section{CONCLUSIONS}

We suggest that critical rehabilitation phases exist and that a failure to exploit the early phase may not be compensated by later rehabilitation. Rehabilitation should be started as soon as possible. In addition, rehabilitation should be both intensive and task specific. Although various aspects of rehabilitation are important, gait rehabilitation seems crucial because of its association with other functional abilities. In the future, longitudinal studies should compare the long-term effects of 
intensive and conventional treatments, including calculations of cost-effectiveness.

\section{ACKNOWLEDGMENTS}

We thank the physicians in the Department of Neurology, Kuopio University Hospital, and Pirjo Huuskonen, PT, for their help during the data collection and Vesa Kiviniemi, PhLic, University of Kuopio, for statistical assistance.

This material was based on work supported by the Brain Research and Rehabilitation Center Neuron, Kuopio, Finland, and the Department of Neurology, University of Kuopio, and Kuopio University Hospital, Kuopio, Finland (grant EVO477338, 57/2003, 36/2004).

The authors have declared that no competing interests exist. The Gait Trainer was purchased at its regular retail price, and none of the authors has any financial interest in the company manufacturing the equipment.

\section{REFERENCES}

1. Jørgensen HS, Nakayama H, Raaschou HO, Olsen TS. Stroke. Neurologic and functional recovery: The Copenhagen Stroke Study. Phys Med Rehabil Clin N Am. 1999; 10(4):887-906. [PMID: 10573714]

2. Jørgensen HS, Nakayama H, Raaschou HO, Olsen TS. Recovery of walking function in stroke patients: The Copenhagen Stroke Study. Arch Phys Med Rehabil. 1995; 76(1):27-32. [PMID: 7811170]

3. Visintin M, Barbeau H, Korner-Bitensky N, Mayo NE. A new approach to retrain gait in stroke patients through body weight support and treadmill stimulation. Stroke. 1998; 29(6):1122-28. [PMID: 9626282]

4. Kosak MC, Reding MJ. Comparison of partial body weight-supported treadmill gait training versus aggressive bracing assisted walking post stroke. Neurorehabil Neural Repair. 2000;14(1):13-19. [PMID: 11228945]

5. Nilsson L, Carlsson J, Danielsson A, Fugl-Meyer A, Hellstrom K, Kristensen L, Sjölund B, Sunnerhagen KS, Grimby G. Walking training of patients with hemiparesis at an early stage after stroke: A comparison of walking training on a treadmill with body weight support and walking training on the ground. Clin Rehabil. 2001;15(5):515-27. [PMID: 11594641$]$

6. Da Cunha IT Jr, Lim PA, Qureshy H, Henson H, Monga T, Protas EJ. Gait outcomes after acute stroke rehabilitation with supported treadmill ambulation training: A random- ized controlled pilot study. Arch Phys Med Rehabil. 2002; 83(9):1258-65. [PMID: 12235606]

7. Schauer M, Mauritz KH. Musical motor feedback (MMF) in walking hemiparetic stroke patients: Randomized trials of gait improvement. Clin Rehabil. 2003;17(7):713-22. [PMID: 14606736]

8. Bach-y-Rita P. Brain plasticity as a basis of the development of rehabilitation procedures for hemiplegia. Scand J Rehabil Med. 1981;13(2-3):73-83. [PMID: 7345569]

9. Duncan PW, Zorowitz R, Bates B, Choi JY, Glasberg JJ, Graham GD, Katz RC, Lamberty K, Reker D. Management of adult stroke rehabilitation care: A clinical practice guideline. Stroke. 2005;36(9):e100-143. [PMID: 16120836]

10. Cifu DX, Stewart DG. Factors affecting functional outcome after stroke: A critical review of rehabilitation interventions. Arch Phys Med Rehabil. 1999;80(5 Suppl 1):S35-39. [PMID: 10326901]

11. Ottenbacher KJ, Jannell S. The results of clinical trials in stroke rehabilitation research. Arch Neurol. 1993;50(1):37-44. [PMID: 8418798]

12. Parry RH, Lincoln NB, Vass CD. Effect of severity of arm impairment on response to additional physiotherapy early after stroke. Clin Rehabil. 1999;13(3):187-98.

[PMID: 10392645]

13. Paolucci S, Antonucci G, Grasso MG, Morelli D, Troisi E, Coiro P, Bragoni M. Early versus delayed inpatient stroke rehabilitation: A matched comparison conducted in Italy. Arch Phys Med Rehabil. 2000;81(6):695-700.

[PMID: 10857508]

14. Wade DT, Collen FM, Robb GF, Warlow CP. Physiotherapy intervention late after stroke and mobility. BMJ. 1992; 304(6827):609-13. [PMID: 1559090]

15. Kwakkel G, Van Peppen R, Wagenaar RC, Wood-Dauphinee S, Richards C, Ashburn A, Miller K, Lincoln N, Partridge C, Wellwood I, Langhorne P. Effects of augmented exercise therapy time after stroke: A meta-analysis. Stroke. 2004; 35(11):2529-39. [PMID: 15472114]

16. Tarkka IM, Pitkänen K, Sivenius J. Paretic hand rehabilitation with constraint-induced movement therapy after stroke. Am J Phys Med Rehabil. 2005;84(7):501-5. [PMID: 15973086]

17. Kwakkel G, Wagenaar RC, Twisk JW, Lankhorst GJ, Koetsier JC. Intensity of leg and arm training after primary middle-cerebral-artery stroke: A randomised trial. Lancet. 1999; 354(9174):191-96. [PMID: 10421300]

18. Kwakkel G, Wagenaar RC. Effect of duration of upper- and lower-extremity rehabilitation sessions and walking speed on recovery of interlimb coordination in hemiplegic gait. Phys Ther. 2002;82(5):432-48. [PMID: 11991797]

19. Van Swieten JC, Koudstaal PJ, Visser MC, Schouten HJ, Van Gijn J. Interobserver agreement for the assessment of 
JRRD, Volume 44, Number 5, 2007

handicap in stroke patients. Stroke. 1988;19(5):604-7. [PMID: 3363593$]$

20. Holden MK, Gill KM, Magliozzi MR, Nathan J, PiehlBaker L. Clinical gait assessment in the neurologically impaired. Reliability and meaningfulness. Phys Ther. 1984; 64(1):35-40. [PMID: 6691052]

21. Mahoney FI, Barthel DW. Functional evaluation: The Barthel Index. Md State Med J. 1965;14:61-65. [PMID: 14258950]

22. De Haan R, Horn J, Limburg M, Van Der Meulen J, Bossuyt P. A comparison of five stroke scales with measures of disability, handicap, and quality of life. Stroke. 1993;24(8):1178-81. [PMID: 8342193]

23. MacKay-Lyons M, Makrides L, Speth S. Effect of $15 \%$ body weight support on exercise capacity of adults without impairments. Phys Ther. 2001;81(11):1790-1800. [PMID: 11694172]

24. Colby SM, Kirkendall DT, Bruzga RF. Electromyographic analysis and energy expenditure of harness supported treadmill walking: Implications for knee rehabilitation. Gait Posture. 1999;10(3):200-205. [PMID: 10567751]

25. Hesse S, Helm B, Krajnik J, Gregoric M, Mauritz KH. Treadmill training with partial body weight support: Influence of body weight release on the gait of hemiparetic patients. J Neurol Rehabil. 1997;11(1):15-20.

26. Hesse S, Werner C, Paul T, Bardeleben A, Chaler J. Influence of walking speed on lower limb muscle activity and energy consumption during treadmill walking of hemiparetic patients. Arch Phys Med Rehabil. 2001;82(11): 1547-50. [PMID: 11689974$]$
27. Danielsson A, Sunnerhagen KS. Oxygen consumption during treadmill walking with and without body weight support in patients with hemiparesis after stroke and in healthy subjects. Arch Phys Med Rehabil. 2000;81(7):953-57. [PMID: 10896011]

28. Barbeau H, Visintin M. Optimal outcomes obtained with body-weight support combined with treadmill training in stroke subjects. Arch Phys Med Rehabil. 2003;84(10): 1458-65. [PMID: 14586912]

29. Borg GA. Psychophysical bases of perceived exertion. Med Sci Sports Exerc. 1982;14(5):377-81. [PMID: 7154893]

30. Wade DT. Measurement in neurological rehabilitation. Oxford (England): Oxford University Press; 1992. p. 388.

31. Guyatt GH, Pugsley SO, Sullivan MJ, Thompson PJ, Berman L, Jones NL, Fallen EL, Taylor DW. Effect of encouragement on walking test performance. Thorax. 1984; 39(11): 818-22. [PMID: 6505988]

32. Collen FM, Wade DT, Bradshaw CM. Mobility after stroke: Reliability of measures of impairment and disability. Int Disabil Stud. 1990;12(1):6-9. [PMID: 2211468]

33. Carr JH, Shepherd RB, Nordholm L, Lynne D. Investigation of a new motor assessment scale for stroke patients. Phys Ther. 1985;65(2):175-80. [PMID: 3969398]

34. Richards CL, Malouin F, Wood-Dauphinee S, Williams JI, Bouchard JP, Brunet D. Task-specific physical therapy for optimization of gait recovery in acute stroke patients. Arch Phys Med Rehabil. 1993;74(6):612-20. [PMID: 8503751$]$

Submitted for publication May 4, 2006. Accepted in revised form June 22, 2007. 